\title{
SIMULATIONS OF ELECTRON INJECTION INTO PLASMA WAKE FIELDS BY COLLIDING LASER PULSES USING XOOPIC
}

\author{
R. E. Giacone, CIPS University of Colorado at Boulder \\ J. R. Cary, CIPS University of Colorado and Tech-X Corporation \\ D. Bruhwiler, Tech-X Corporation \\ E. Esarey, W. Leemans, Lawrence Berkeley National Laboratory \\ P. Mardahl, J. P. Verboncoeur, University of California at Berkeley
}

\begin{abstract}
First time, self-consistent particle-in-cell simulations of electron injection into a plasma wake field by colliding laser pulses are presented. This method for injecting electrons in a laser wake field has been proposed recently by Esarey and coworkers [1]. An intense pump pulse generates a fast wake field. Forward going and backward going injection pulses collide at some distance behind the pump pulse generating a slow ponderomotive beat wave which can be used to inject plasma electrons into the fast wake field for acceleration to high energies. We have modified XOOPIC [2] to run numerical simulations of the colliding pulses scheme. We have analyzed the influence of plasma and pulses parameters, so that we are able to study electron injection as it varies with amplitudes, wavelengths, and phases of the pulses. We will present preliminary results.
\end{abstract}

\section{INTRODUCTION}

Plasma-based accelerators have received much theoretical and experimental attention in the last decade due to the large longitudinal electric fields that can be excited in a plasma without the limitations found in conventional accelerators. One widely investigated and very promising concept is the laser wakefield accelerator (LWFA). Recently, a new method for injecting electrons in a LWFA has been proposed [1] that uses two laser pulses, which propagate parallel to one another. When the injection pulses collide, a slow phase velocity beat wave is generated that injects electrons into the fast wake field for acceleration. Some preliminary theoretical analysis and simulations in onedimensional space have been done to study the colliding laser pulses scheme [7], but to fully understand the complex phenomena involved, full two-dimensional scale simulations are required.

The rest of the paper is organized as follows: in Sec. 2 a brief description of XOOPIC code with the modifications we made is given. In Sec. 3 we described the injection laser scheme. In Sec. 4 we present the results of the simulations, and summary and conclusions are presented in Sec. 5 .

\section{THE XOOPIC CODE}

XOOPIC is an object-oriented two-dimensional relativistic electromagnetic particle-in-cell code written in C++ by J. P. Verboncoeur and collaborators at the University of
California at Berkeley [2]. The XOOPIC code has implemented Cartesian and Cylindrical geometries. It can handle electrostatic and electromagnetic simulations and relativistic and non-relativistic particles The code also has an implementation to run on MPI-based parallel machines. The particles are advanced using the relativistic time-center Boris scheme [3]. The code uses a charge conserving current weighting algorithm, which ensures that Gauss' law remains satisfied if it was initially satisfied.

The nature of the problems we want to address necessitate an understanding of the evolution of an intense short laser pulse propagating through a plasma. Because only the region of the plasma near the laser pulse is of interest, we need to follow the evolution of the laser pulse using a computational moving window which moves with the laser pulse at approximately the speed of light. We also need the code to allow launching a Gaussian electromagnetic wave from a boundary to simulate a laser beam entering the plasma. Neither of these two requirements were implemented in the original version of XOOPIC, therefore our first task was to modify XOOPIC to include these capabilities.

\section{LASER WAKE FIELD ACCELERATOR SCHEMES}

The accelerating electric field (or gradient) in conventional radio frequency linear accelerators is limited to around $100 \mathrm{MV} / \mathrm{m}$, partly due to heating or breakdown on the walls of the structures. In order to accelerate electrons to very high energies (greater than $1 \mathrm{TeV}$ ), it is necessary to develop new acceleration concepts providing a higher electric field. Plasmas can support large high longitudinal electric fields. More precisely, ionized plasmas can sustain electron plasma waves (EPW) with longitudinal electric field on the order of the nonrelativistic wavebreaking field [4], $E_{0}=c m_{e} \omega_{p} / e$. For an electron density of $n_{e}=10^{18} \mathrm{~cm}^{-3}$ the electric field is $E_{0} \approx 100 \mathrm{GV} / \mathrm{m}$ (which is approximately three orders of magnitude greater that obtained in conventional RF linacs) with a phase velocity close to the speed of light.

In the laser wakefield accelerator (LWFA), a single short $(\leq 1 \mathrm{ps}$.$) , ultrahigh intensity \left(\geq 10^{18} \mathrm{~W} / \mathrm{cm}^{2}\right)$ laser pulse injected in an underdense plasma excites an EPW behind the pulse. The plasma wake is excited by the ponderomotive force created by the photons. A correctly placed trail- 
ing electron bunch can be accelerated by the longitudinal electric field and focused by the transverse electric field of the plasma wake. Although several recent experiments [5] - [6] have demonstrated the self-trapping and acceleration of electrons, the production of electrons beams with low momentum spread and good pulse-to-pulse energy stability will require injection of ultrashort electron bunches into the wake field with femtosecond timing accuracy. These requirements are beyond the current state-of-the art performance of photocathode radio-frequency electron guns [1].

Recently a method for injecting electrons in a LWFA has been proposed [1] that uses two laser pulses which propagate either perpendicular or parallel to one another. The colliding pulse scheme uses three short laser pulses (Fig .1). An intense pump pulse generates a fast $\left(v_{p 0} \approx c\right)$ wake field. A forward going and a backward going injection pulses collide at some distance behind the pump pulse generating a slow ponderomotive beat wave with phase velocity $v_{p b} \approx \Delta \omega / 2 k_{0}$. During the time in which the two injection pulses overlap, the slow beat wave injects plasma electrons into the fast wake field for acceleration to high energies. Injection and acceleration can occur at low densities $\left(\lambda_{p} / \lambda \approx 100\right)$, thus allowing for high single-stage energy gains. The colliding pulse scheme offers detailed control of the injection process. The injection phase can be controlled via the position of the forward injection pulse, the beat phase via $\Delta \omega$, the injection energy via the pulse amplitude, and the number of trapped electrons via the backward pulse duration.

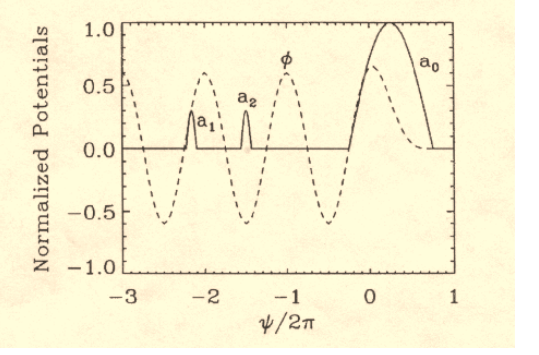

Figure 1: Profiles of the pump laser pulse $a_{0}$, the wake $\phi$, and the forward $a_{1}$ injection pulse, all of which are stationary in the $\psi=k_{p}\left(x-v_{p 0} t\right)$ frame, and the backward injection pulse $a_{2}$, which moves to the left at $\approx 2 c$ [1].

\section{SIMULATIONS RESULTS}

We initially began our investigation trying to understand and reproduce the results found in Schroeder's paper. We set trial runs using the same plasma and pulses parameters as the ones used in that paper, but our simulations showed some discouraging results. We observed particles got trapped and were accelerated forming a very low quality beam. After a quarter Rayleigh length of propagation the beam had $100 \%$ energy spread and shortly after that all particles were lost. It was clear that particles were not being injecting at the right point in phase space to keep them trapped into the plasma wake. Our analysis showed that increasing the intensity of the injection pulses will put the particles into a region in phase-space where there can be accelerated and focused at the same time. Thus, we used Schroeder's paper parameters as a reference but modified the intensity of the injection pulses as well as the point in space where the pulses collide. The electron plasma density was $n_{e 0}=6.9 \times 10^{17} \mathrm{~cm}^{-3}$ which corresponds to a wavelength of $\lambda_{p}=40 \mu \mathrm{m}$ and to a plasma frequency $\omega_{p}=4.7 \times 10^{13} s^{-1}$. The laser pulses were linearly polarized with transverse Gaussian profile. The minimum laser spot size was $15 \mu \mathrm{m}$ and the Rayleigh length was about $22 \lambda_{p}$. The laser pulse length was chosen to be about $\lambda_{p}$. The pump laser intensity was $I_{L}=3.29 \times 10^{18} \mathrm{~W} / \mathrm{cm}^{2}$ and the laser wavelength $\lambda_{0}=0.8 \mu \mathrm{m}$. The forward injection laser pulse wavelength was $\lambda 1=0.83 \mu \mathrm{m}$ and intensity $I_{L}=1.7 \times 10^{18} \mathrm{~W} / \mathrm{cm}^{2}$, the backward pulse had $\lambda_{2}=0.8 \mu \mathrm{m}$ and intensity $I_{L}=1.9 \times 10^{18} \mathrm{~W} / \mathrm{cm}^{2}$. The pulse length was $\lambda_{p} / 2$ for both injection pulses.

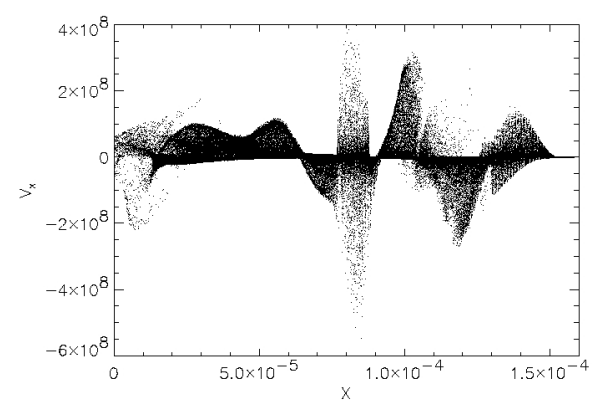

Figure 2: Snapshot of electrons in $X-V_{x}$ phase-space during collision of injection pulses.

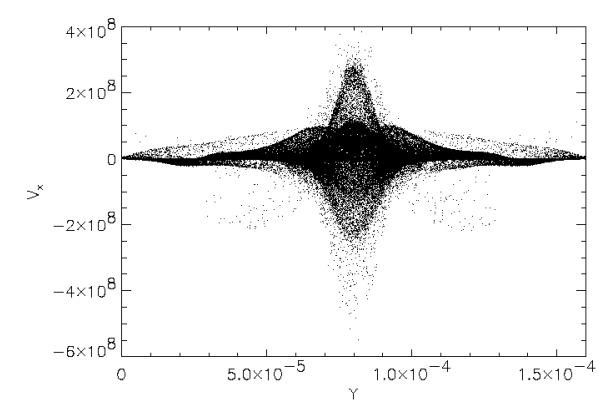

Figure 3: Snapshot of electrons in $Y-V_{x}$ phase-space during collision of injection pulses.

The length of the simulation box was $L_{x}=160 \mu \mathrm{m}$ in both, $X$ and $Y$ directions. The computational mesh consisted of 4000 cells in the $X$ direction and 300 cells in the $Y$ direction. The simulation used about 4,800,000 computational particles.

The pump pulse is launched into a vacuum region of 20 $\mu \mathrm{m}$. It follows a $(1-$ cosine $)$ rise density of $60 \mu \mathrm{m}$, and then a flat density region of $60 \mu \mathrm{m}$. At $t=0$ the pump pulse 
is launched. At $c t=65 \mu \mathrm{m}$ the forward pulse is launched from the left boundary. At $c t=130 \mu m$ the backward pulse is launched from the right boundary. At $c t=155 \mu \mathrm{m}$, the moving window is turned on. The injection pulses collided at $c t=185 \mu \mathrm{m}$. Figures 2 to 7 are snapshots of electrons in $X-V_{x}$ and $Y-V_{x}$ phase-space at different stages during the collision of the injection pulses. We observed that during the collision (Fig. 2), the beating of the injection laser pulses picks up some electrons from the background plasma. After half Rayleigh length of propagation (Fig. 4) one can see two bunches getting trapped. The one at the front got trapped without being injecting by the colliding pulses. Because those particles were randomly picked up from the background most of them will fall into a defocusing region and will eventually get lost as the propagation continues. The second bunch looks more beam-like. These are the particles injected at the right point in phase-space by the injection pulses. Fig. 6 shows the particles after a Rayleigh length of propagation.

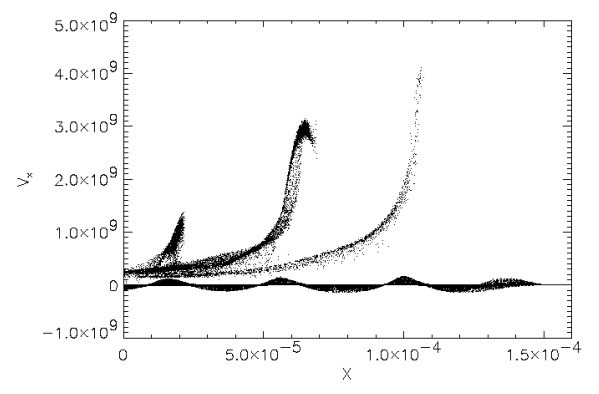

Figure 4: Snapshot of electrons in $X-V_{x}$ phase-space after about half Rayleigh length of propagation.

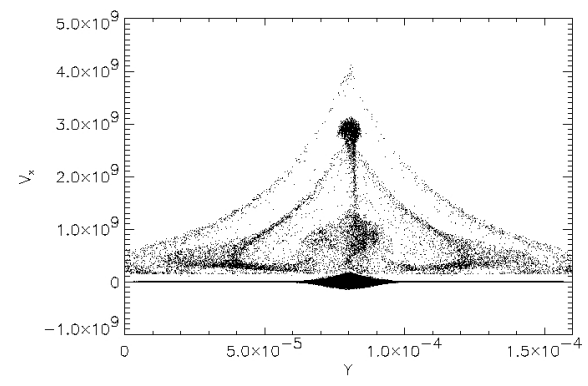

Figure 5: Snapshot of electrons in $Y-V_{x}$ phase-space after about Rayleigh length of propagation.

One can see particles which where in a defocusing region (which are at greater radii) lost their energy. On the contrary, particles in the focusing region form a nice beam (Fig. 7). The faster particles are at a relativistic factor of $\gamma=31$ in about $700 \mu m$, which corresponds to an accelerating gradient of $21 \mathrm{GeV} / \mathrm{m}$. The quality of the beam is also good. The emittance defined as $\sigma_{v_{y}} \sigma_{y} /<v_{x}>$ is 0.017 and $\sigma_{v_{x}}=0.71 \mathrm{MeV}$.

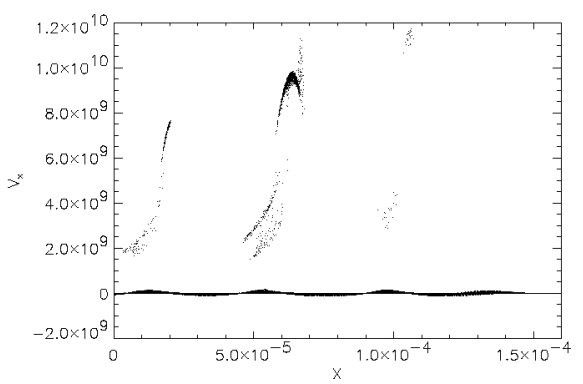

Figure 6: Snapshot of electrons in $X-V_{x}$ phase-space after a Rayleigh length of propagation.

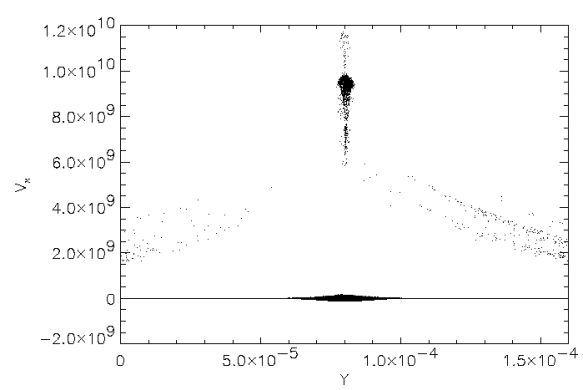

Figure 7: Snapshot of electrons in $Y-V_{x}$ phase-space after a Rayleigh length of propagation.

\section{CONCLUSIONS}

We have presented the first time self-consistent PIC simulations of electron injection into a plasma wake field by colliding laser pulses. We made use of an existing proven code, XOOPIC and introduced new capabilities (electromagnetic wave launcher, parallel implementation of moving window) to to allow simulations of laser-plasma interaction processes. The results of our simulations show that after one Rayleigh length of propagation a good quality beam is produced.

\section{REFERENCES}

[1] E. Esarey, R. F. Hubbard, W. P. Leemans, A. Ting and P. Sprangle, " Phys. Rev. Lett 79, 2682 (1997).

[2] J. P. Verboncoeur, A. B. Langdon and N. T. Gladd, Phys. Comm 87, 199 (1995).

[3] C. K. Birdsall and A. B. Langdon, "Plasma Physics via Computer Simulations" (Adam Hilger, Bristol, 1991).

[4] E. Esarey, P. Sprangle, J. Krall and A. Ting, "IEEE Trans. Plasma Sci.” 24, 252 (1996).

[5] K. Nakajima, "Phys. Rev. Lett." 74, 4428 (1995).

[6] A. Ting, "Phys. Plasmas" 4, 1889 (1997).

[7] C. B. Schroeder, P. B. Lee, J. S. Wurtele, E. Esarey and W. P. Leemans, "Phys. Rev. E” 59, 6037 (1999). 